\title{
MicroRNA-33b-5p is overexpressed and inhibits GLUT4 by targeting HMGA2 in polycystic ovarian syndrome: An in vivo and in vitro study
}

\author{
YING YANG $^{1 *}$, HUA JIANG ${ }^{2 *}$, LING XIAO $^{3}$ and XUEZHOU YANG ${ }^{4}$
}

\begin{abstract}
Departments of ${ }^{1}$ Endocrinology and ${ }^{2}$ Clinical Laboratory, Xiangyang Central Hospital, Affiliated Hospital of Hubei University of Arts and Science; ${ }^{3}$ Department of Gynecology, Xiangyang Maternal and Child Health Care Hospital;

${ }^{4}$ Center for Reproductive Medicine, Xiangyang Central Hospital, Affiliated Hospital of Hubei University of Arts and Science, Xiangyang, Hubei 441021, P.R. China
\end{abstract}

Received July 20, 2017; Accepted April 3, 2018

DOI: $10.3892 /$ or.2018.6375

\begin{abstract}
Polycystic ovary syndrome (PCOS) is a common endocrine and metabolic disease, but its pathogenesis remains largely unknown. The present study explored the role of microRNA-33b-5p (miR-33b-5p) in PCOS pathogenesis, with a particular focus on its role in regulating glucose transporter 4 (GLUT4). A rat model of PCOS was developed by injecting female SD rats with insulin and HCG. miR-33b-5p, GLUT4, sterol regulatory element-binding protein 1 (SREBF1), and high mobility group A2 (HMGA2) expression in rat ovarian tissues was examined by qRT-PCR and immunohistochemistry. The effect of a high dose of either glucose or insulin on miR-33b-5p, GLUT4, SREBF1 and HMGA2 expression was also examined in cultured adipocytes by qRT-PCR and western blotting. Additionally, the luciferase reporter assay and chromatin immunoprecipitation (ChIP) were used to explore the role of miR-33b-5p in regulating HMGA2, SREBF-1 and/or GLUT4. Elevated levels of miR-33b-5p expression were detected in the ovarian tissues of insulin resistant PCOS rats, and those levels were negatively correlated with those of GLUT4, HMGA2 and SREBF1 expression $(\mathrm{P}<0.05)$. Immunohistochemistry studies revealed that GLUT4, SREBF1, and HMGA2 expression levels in the ovarian tissues of insulin resistant PCOS rats were significantly lower than those in other groups of rats. In cultured
\end{abstract}

Correspondence to: Dr Xuezhou Yang, Center for Reproductive Medicine, Xiangyang Central Hospital, Affiliated Hospital of Hubei University of Arts and Science, Jingzhou Street 136, Xiangcheng, Xiangyang, Hubei 441021, P.R. China

E-mail:1vv06113@163.com

${ }^{*}$ Contributed equally

Key words: polycystic ovary syndrome, microRNA-33b-5p, SREBF1, GLUT4, HMGA2 adipocytes, excess extracellular glucose or insulin increased miR-33b-5p expression but reduced GLUT4, SREBF1 and HMGA2 expression, whereas the levels of GLUT4, SREBF1 and HMGA2 were elevated by inhibition of miR-33b-5. HMGA2 could directly bind to the 5'-promoter region of GLUT4 and promote its expression, and could also promote SREBF1 expression. Moreover, SREBF1 could also directly bind to the 5'-promoter region of GLUT4 and promote its expression. Our findings revealed that miR-33b-5p was overexpressed in the ovarian tissues of insulin resistant PCOS rats, and thus may play an important role in the development of insulin resistance in PCOS patients. miR-33b-5p can inhibit GLUT4 production by targeting $H M G A 2$, and in addition, HMGA2 and SREBF1 are important molecules involved in modulating GLUT4 expression.

\section{Introduction}

Polycystic ovary syndrome (PCOS) is a common endocrine disease affecting 5-20\% of women of reproductive age worldwide (1). PCOS is characterized by high luteinizing hormone levels, polycystic ovarian morphology, hyperandrogenism, hyperinsulinemia, and insulin resistance. Excessive androgen production by the ovaries is the main feature of $\operatorname{PCOS}(1,2)$. The prevalence of PCOS has increased during the past decade, and its adverse effects on the health of women are becoming more serious $(3,4)$. Moreover, women with PCOS are at increased risks for cardiovascular disease and diabetes (5-7). Therefore, when considering its increasing prevalence, more effective methods for preventing or treating PCOS are needed. Presently, the pathogenesis of PCOS is not fully understood, and requires further investigation. More than $50 \%$ of PCOS patients are insulin resistant $(2,8)$, which can partly explain their increased risk for diabetes and cardiovascular disease. Furthermore, insulin resistance can also be the cause for hyperlipidemia and hyperinsulinemia in PCOS patients. Therefore, identifying key factors involved in the development of insulin resistance should provide us with a better understanding of PCOS pathogenesis. For example, glucose transporter 4 (GLUT4) is a protein that plays critical roles in 
controlling the translocation of glucose into adipocytes and regulating glucose homeostasis $(9,10)$. GLUT4 is strongly associated with insulin sensitivity, and its dysfunction has been reported to cause insulin resistance in PCOS patients (11-13). High mobility group A2 (HMGA2), a non-histone chromosomal protein, is associated with lipomas, suggesting its roles in adipogenesis and insulin resistance $(14,15)$. SREBF1, also known as sterol regulatory element-binding protein 1 (SREBP1), is a major regulator of genes involved in metabolic pathways and sterol biosynthesis (16-18), and its expression is significantly increased in the endometrium of PCOS patients (17). More importantly, the transcripts for the two hsa-miR-33 isoforms ( $a$ and $b$ ) are located in a non-coding region of the $S R E B F$ gene, suggesting a potential role for miR-33 in regulating SREBF1 expression (19). However, the underlying molecular mechanisms that regulate these key factors and lead to insulin resistance in PCOS patients remain poorly understood.

MicroRNAs (miRNAs) consist of a group of small non-coding RNAs $20-24$ nt in length (20). Previous studies have revealed that miRNAs play key roles in modulating the functions of human bodies (20-22). miRNAs mainly exert their effects by binding to the 3'-untranslated regions (3'-UTRs) of target genes and inhibiting transcription of the mRNAs of those genes (22). Recent studies have proposed that miRNAs are important gene regulators involved in human diseases, including endocrine and metabolic diseases $(23,24)$. Some miRNAs have been found to play critical roles in modulating glucose homeostasis, and the aberrant expression of those miRNAs can result in insulin resistance that is often involved in the development of metabolic diseases $(25,26)$. Several miRNAs (i.e. miR-93 and miR-320) have been implicated in the pathogenesis of PCOS (27-32). However, our understanding of their involvement in the development of PCOS is limited, and more studies are needed to clarify their roles in PCOS pathogenesis. In the present study, we examined the levels of miR-33b-5p expression in the ovarian tissues of PCOS rats with insulin resistance, and the correlations between miR-33b-5p levels and the expression of three different proteins: GLUT4, HMGA2 and SREBF1. We also investigated the possible role of miR-33b-5p in regulating GLUT4, HMGA2 and SREBF1 in cultured adipocytes.

\section{Materials and methods}

Animal model of PCOS. Twenty-four female Sprague-Dawley (SD) rats (180-250 g) were obtained from the Center of Laboratory Animal Science, Hubei, China. The rats were group-housed in a room with a regular 12-h light/dark cycle, and had free access to food and water. All animals were treated in accordance with the guidelines of the National Institutes of Health Guide for the Care and Use of Laboratory Animals, and the study protocol was approved by the Animal Ethics Committee of Hubei University of Arts and Science.

The PCOS model was developed in female rats according to the method described by Poretsky et al (33). Female rats ( 70 to 85 days old) were subcutaneously injected with increasing doses of insulin (0.5 IU, 5.0 IU and 5.0 IU/day) for the first 10 days, and then with 5 IU insulin + 1.5 IU HCG from day 11 through day 22. The rats were fed a high fat diet for 6 weeks, and their weights were measured weekly. Rats in the control groups were fed a normal diet every day. Finally, the PCOS model rats displayed typical characteristics of PCOS, such as hyperinsulinemia, insulin resistance, weight gain, and polycystic degeneration in the ovaries (33). Fasting blood glucose and fasting insulin levels were assessed and the homeostatic model assessment-insulin resistance (HOMA-IR) of each rat was calculated. Next, the rats were assigned to four different groups: a non-PCOS/non-IR group, a non-PCOS/IR group, a PCOS/non-IR group, and a PCOS/IR group. The rats were then anesthetized with $10 \%$ chloral hydrate, and laparotomies were performed to collect ovarian tissue specimens for further processing. Insulin resistance was assessed by the HOMA-IR index, and rats with a HOMA-IR $\geq 2.5$ were considered insulin resistant (IR).

Hematoxylin and eosin $(H \& E)$ and immunohistochemical staining. The ovaries of each rat were removed, processed, and sectioned at 4-mm thickness; after which, the sections were stained with $H \& E$ and examined for evidence of morphological changes. Five sections were obtained from each ovary. Immunohistochemistry (IHC) was then used to examine the ovarian tissues for SREBF1, HMGA2 and GLUT4 expression. Tissue sections used for immunohistochemistry were first deparaffinized and hydrated, and endogenous peroxidase activity was quenched with $0.3 \%$ hydrogen peroxide in PBS. Next, the tissue sections were incubated in blocking buffer for $60 \mathrm{~min}$, and then incubated overnight at $4^{\circ} \mathrm{C}$ with primary antibodies that included GLUT4 antibody (1:500; rabbit polyclonal; cat. no. PB0143; Boster Biological Technology, Wuhan, China), SREBF1 antibody (1:500; rabbit polyclonal; cat. no. 14088-1-AP; Proteintech; Wuhan Sanying Biotechnology, Wuhan, China), and HMGA2 antibody (1:500; rabbit polyclonal; cat. no. bs-0556R; Bioss Antibodies, Beijing, China). They were then incubated with horseradish peroxidase-conjugated secondary antibodies [1:5,000; goat anti-rabbit IgG H\&L (HRP); cat. no. ab205718; Abcam, Cambridge, MA, USA] as per the manufacturer's instructions. Positively-labeled cells were visualized using a streptavidin-HRP conjugate and DAB chromogen. The tissue sections were then counterstained with hematoxylin, dehydrated, and mounted in DPX.

Culturing of human adipocytes in high glucose medium. Human adipocyte cells (ScienCell Research Laboratories, Inc., San Diego, CA, USA) were incubated with adipocyte maintenance medium at $37^{\circ} \mathrm{C}$ in a humidified incubator containing 5-10\% $\mathrm{CO}_{2}$. The adipocyte cells were then cultured in normal adipocyte maintenance medium (control group) or treated with different concentrations of high-glucose adipocyte maintenance medium (Gibco; Thermo Fisher Scientific, Inc., Waltham, MA, USA) $(0,3.15,9.45$ or $28.35 \mathrm{~g} / \mathrm{l})$ for $24 \mathrm{~h}$. After culture, the adipocytes were collected and their levels of miR-33b-5p, SREBF1, HMGA2 and GLUT4 were examined using real-time polymerase chain reaction (PCR) or and western blotting methods.

Culturing of human adipocytes in different concentrations of insulin. Human adipocytes were incubated with adipocyte maintenance medium at $37^{\circ} \mathrm{C}$ in a humidified incubator 
Table I. PCR primer information.

Sequence (5'-3')

Product

ID

CTCGCTTCGGCAGCACA

U6 F

AACGCTTCACGAATTTGCGT

U6 R

CCTCGTCTCATAGACAAGATGGT

169

r-GAPDH F

GGGTAGAGTCATACTGGAACATG

h-GAPDH F

TGTTCGTCATGGGTGTGAAC

154

h-GAPDH R

ATGGCATGGACTGTGGTCAT

r-miR-33-5p RT

r-miR-33-5p F

CTCAACTGGTGTCGTGGAGTC GGCAATTCAGTTGAGTGCAATGC

ACACTCCAGCTGGGGTGCATTGTAGTTGCAT

h-miR-33b-5p RT

h-miR-33b-5p F

CTCAACTGGTGTCGT GGAGTCGGCAATTCAGTTGAGGCAATGCA
ACACTCCAGCTGGGGTGCATTGCTGTTGCA

ALL R

CTCAACTGGTGTCGTGGA

r-SREBF1 F

TTACAGCACAGCAACCAGAA

132

r-SREBF1 R

CATGCCCTCCATAGACACAT

h-SREBF1 F

GCAACACAGCAACCAGAAACT

191

h-SREBF1 R

GGAAAGGTGAGCCAGCATC

r-HMGA2 F

CCTGTGAGCCCTCTCCTAA

CCGTTTTTCTCCAATGGTC

r-HMGA2 R

AGCAGCAGCAAGAACCAAC

length (bp)

h-HMGA2 F

h-HMGA2 R

CCCAGGCAAGGCAACAT

r-GLUT4 F

TGGTTGGGAAGGAAAAGG

107

AGATGAGTGGGGGCGAT

r-GLUT4 R

GCCCCCGCTACCTCTACAT

CCGCTCCACCAACAAC

h-GLUT4 R

r, rats; h, humans; F, forward; R, reverse; RT, reverse transcription.

containing 5-10\% $\mathrm{CO}_{2}$. The adipocyte cells were then treated with different doses of insulin (Sigma-Aldrich, Merck KGaA, Darmstadt, Germany) (0, 5, 50 or $500 \mathrm{nM})$ for $24 \mathrm{~h}$; after which, they were collected and tested for their levels of miR-33b-5p, SREBF1, HMGA2 and GLUT4 expression using real-time PCR or western blotting methods.

Treatment of human adipocytes with different concentrations of $m i R-33 b-5 p$ inhibitor. Human adipocytes were cultured as aforementioned; after which, they were seeded into 6-well plates and grown to $60-80 \%$ confluence for use in transfection studies. A 9- $\mu 1$ volume of Lipofectamine 2000 was mixed with $150 \mu \mathrm{l}$ of Opti-MEM medium (both from Thermo Fisher Scientific, Inc.). A miR-33b-5p inhibitor or NC inhibitor (Shanghai JiKai Gene Chemical Technology Co., Ltd., Shanghai, China) was diluted with Opti-MEM medium and then mixed with Lipofectamine-Opti-MEM medium at a ratio of 1:1 to achieve miR-33b-5p inhibitor concentrations of 0,25 , 50 and $100 \mathrm{nM}$, respectively. The sequence of miR-33b-5p inhibitor: 5'-GCAAUGCAACAGCAAUGCAC-3'. After allowing the cells to incubate at room temperature for $5 \mathrm{~min}$, the miR-33b-5p inhibitor was added, and the cells were incubated with the different concentrations of miR-inhibitor complex for $48 \mathrm{~h}$. Next, the adipocytes were collected, and their levels of
miR-33b-5p, SREBF1, HMGA2 and GLUT4 were determined using real-time PCR or western blotting methods.

RNA extraction and real-time PCR. A miRACLE isolation kit (Jinfiniti Biotech, LLC, Augusta, GA, USA) was used to extract total RNA from the ovarian tissues of rats and cultured adipocytes. The various cDNAs of mRNAs or miRNAs were synthesized using a cDNA reverse transcription kit. Real-time PCR was carried out using SYBR-Green Supermix and an Applied Biosystems 7300 Real-Time PCR System (Applied Biosystems; Thermo Fisher Scientific, Inc.). The primers used for PCR are listed in Table I. U6 and GAPDH were used as internal control genes. Real-time PCR was performed on target genes under the following conditions: 35 cycles each containing $94^{\circ} \mathrm{C}$ for $30 \mathrm{sec}, 58^{\circ} \mathrm{C}$ for $45 \mathrm{sec}$ and $72^{\circ} \mathrm{C}$ for $35 \mathrm{sec}$. The relative levels of mRNA and miR-33b-5p were calculated using the $2^{-\triangle \Delta C T}$ method.

Western blotting studies. Proteins were extracted from cultured adipocytes, and then separated using 10\% SDS-PAGE. The separated protein bands were transferred onto a PVDF membrane, which was then probed with rabbit monoclonal antibodies against SREBF1 (1:500; rabbit polyclonal; cat. no. 14088-1-AP; Proteintech); HMGA2 (1:1,000; rabbit 
Table II. Serum fasting glucose and fasting insulin levels and HOMA-IR index in the PCOS rats.

\begin{tabular}{llccr}
\hline Groups & $\mathrm{N}$ & Fasting glucose $(\mathrm{mmol} / \mathrm{l})$ & Fasting insulin $(\mathrm{mIU} / \mathrm{l})$ & HOMA-IR \\
\hline PCOS & 10 & $7.05 \pm 0.93^{\mathrm{a}}$ & $27.6 \pm 2.3^{\mathrm{a}}$ & $7.5 \pm 2.7^{\mathrm{a}}$ \\
Control & 10 & $5.51 \pm 0.58$ & $11.6 \pm 1.9$ & $2.7 \pm 0.3^{\mathrm{a}}$ \\
\hline
\end{tabular}

${ }^{\mathrm{a}} \mathrm{P}<0.05$. PCOS, polycystic ovary syndrome; HOMA-IR, homeostatic model assessment-insulin resistance.

polyclonal; cat. no. bs-0556R; Bioss Antibodies); GLUT4 (1:1,000; rabbit polyclonal; cat. no. PB0143; Boster Biological Technology) or GAPDH (1:2,000; mouse monoclonal; cat. no. ab8245; Abcam) as an internal control overnight at $4^{\circ} \mathrm{C}$. After incubation at $37^{\circ} \mathrm{C}$ for $1 \mathrm{~h}$ with horseradish peroxidase-conjugated secondary antibodies (1:5,000; goat polyclonal to HRP; cat. no. ab181658; Abcam), an Immobilon ${ }^{\mathrm{TM}}$ Western Chemiluminescent ECL kit was used to detect bound antibodies. Protein staining intensity was assessed using Image-Pro Plus 6.0 software.

Luciferase reporter assay. Wild-type and mutant type HMGA2 3'-UTRs were amplified and inserted into psiCHECK2 luciferase vectors (Promega Corporation, Madison, WI, USA). Next, the vectors with either the miR-33b-5p mimics or negative mimics (GenePharma Co., Ltd., Shanghai, China) $(1.5 \mathrm{mg})$ were used to transfect 293T cells. Following $48 \mathrm{~h}$ of transfection, cellular luciferase activity was detected with a Microplate reader (Tecan GENios Pro; Tecan Group, Ltd., Mannedorf, Switzerland) according to the manufacturer's protocol.

HMG2 transcription factor binding sites for GLUT4 and SREBF1 5'-promoter luciferase vectors were amplified and inserted into pGL3 luciferase vectors (Promega Corporation). Next, the vectors with either the pcDNA3.0-HMGA2 overexpression plasmid or empty plasmid $(0,0.5,1.5,2.5$ and $5.0 \mu \mathrm{g})$ were transfected into $293 \mathrm{~T}$ cells. Luciferase activity was examined after $48 \mathrm{~h}$ of transfection. SREBF1 transcription factor binding sites were used to establish GLUT4 5'-promoter wild-type and mutant type luciferase vectors. Vectors with either the pcDNA3.0-SREBF1 overexpression plasmid or empty plasmid were transfected into $293 \mathrm{~T}$ cells, and cellular luciferase activity was assessed.

Chromatin immunoprecipitation (ChIP). To analyze the binding of SREBF1/HMGA2 protein to the GLUT4 gene promoter, adipocyte cells transfected with either SREBF1 or HMGA2 antibodies (Abcam) were used for ChIP analyses that were performed using a Pierce Agarose Chip Kit (EpiGentek Group, Inc., Farmingdale, NY, USA). Normal rabbit IgG was used as a negative control. Non-precipitated genomic DNA input was amplified as an input control. After purification, the DNAs were used for PCR analyses that were performed using primers (Sangon Biotech Co., Ltd., Shanghai, China) that encompassed the GLUT4 promoter region. The conditions used for PCR were as follows: denaturation at $95^{\circ} \mathrm{C}$ for $2 \mathrm{~min}$, followed by 40 cycles at $95^{\circ} \mathrm{C}$ for $20 \mathrm{sec}, 58^{\circ} \mathrm{C}$ for $20 \mathrm{sec}$, and $72^{\circ} \mathrm{C}$ for $20 \mathrm{sec}$.
Statistical analysis. All statistical analyses were performed using IBM SPSS Statistics for Windows, version 19.0 (IBM Corporation, Armonk, NY, USA). The levels of miR-33b-5p and gene expression are shown as the mean $\pm \mathrm{SD}$, and differences between groups were analyzed using Student's t-test. One-way ANOVA and Tukey's test were used to evaluate the levels of miR-33b-5p and gene expression in human adipocytes treated with different concentrations of glucose or insulin. The association between miR-33b-5p and SREBF1/HMGA2 expression was assessed using Pearson's correlation test. $\mathrm{P}$-values $<0.05$ were regarded as statistically significant.

\section{Results}

Expression of miR-33b-5p, GLUT4, SREBF1 and HMGA2 in the ovarian tissues of PCOS rats. The PCOS rat model was successfully developed using the method described by Poretsky et al (33). Insulin resistance in PCOS rats was indicated by their significantly increased insulin resistance index (HOMA-IR) values when compared with those of control rats (Table II). Based on their HOMA-IR score, the rats were then further classified into four groups: a non-PCOS/non-IR group, a non-PCOS/IR group, a PCOS/non-IR group, and a PCOS/IR group. The levels of miR-33b-5p expression in the ovarian tissues of non-PCOS/IR rats were significantly higher than those in the ovarian tissues of non-PCOS/non-IR rats $(\mathrm{P}<0.05)$. The PCOS/IR group also exhibited upregulated levels of miR-33b-5p expression when compared with the PCOS/non-IR group (Fig. 1A). Conversely, the levels of HMGA2, SREBF1, and GLUT4 expression in the ovarian tissues of non-PCOS/IR rats were significantly lower than those in the non-PCOS/non-IR rats (both $\mathrm{P}<0.05$ ). PCOS/IR rats had lower HMG2 and GLUT4 levels when compared with PCOS-non-IR rats (Fig. 1B-D). Furthermore, the levels of HMG2 and GLU4 expression were lower in the PCOS/non-IR group than in the non-PCOS/non-IR group. miR-33b-5p levels were negatively associated with SREBF1 and HMGA2 expression in the ovarian tissues of rats in all four groups $(\mathrm{P}<0.01)$, and miR-33b-5p levels were negatively correlated with GLUT4 levels $(\mathrm{P}<0.01)$ (Fig. 1E-G).

H\&E staining of rat ovarian tissues revealed that ovary oocytes in the non-PCOS/ non-IR group were clear, and large numbers of granulosa cells were present. Ovarian tissues in the other three groups contained significantly fewer granulosa cells. The granulosa cells were loosely arranged, and the cortical stromal cells displayed obvious signs of hyperplasia (Fig. 2). Furthermore, immunohistochemistry was conducted to detect and compare the expression of GLUT4, 

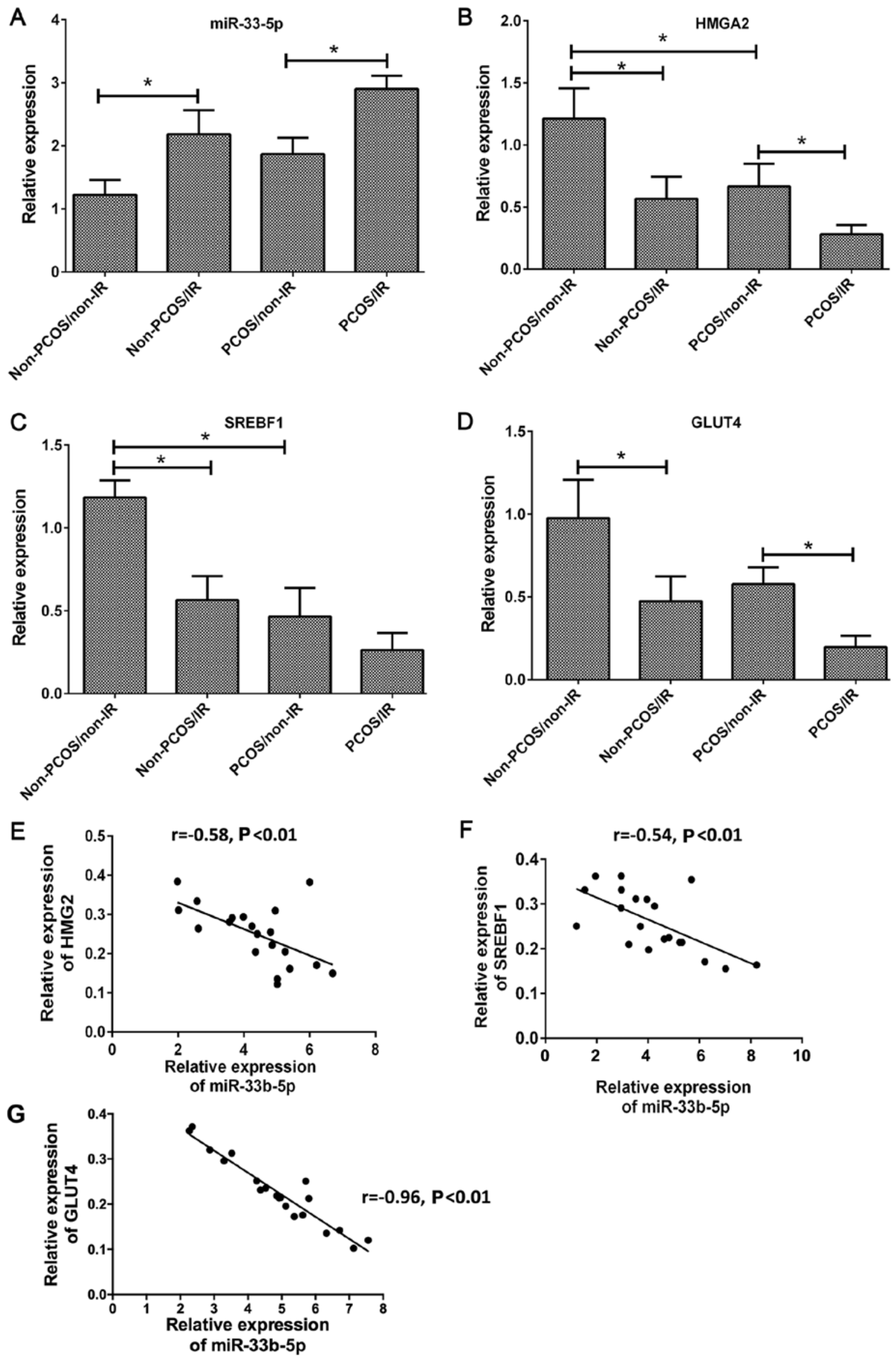

Figure 1. (A-D) The RNA levels and correlations of miR-33b-5p, GLUT4, SREBF1, and HMGA2 in rat ovarian tissues from the non-PCOS/non-IR group, non-PCOS/IR group, PCOS/non-IR group, and PCOS/IR group (U6 and GAPDH were used as internal controls for miR-33b-5p and the genes aforementioned, respectively. Relative expression levels of mRNAs and miRNAs were calculated using the $2^{-\Delta \Delta C T}$ method). (E-G) The respective correlations between miR-33b-5p and HMGA2, SREBF1 and GLUT4 in rat ovarian tissues, ${ }^{*} \mathrm{P}<0.05$. PCOS, polycystic ovary syndrome; IR, insulin resistance. 

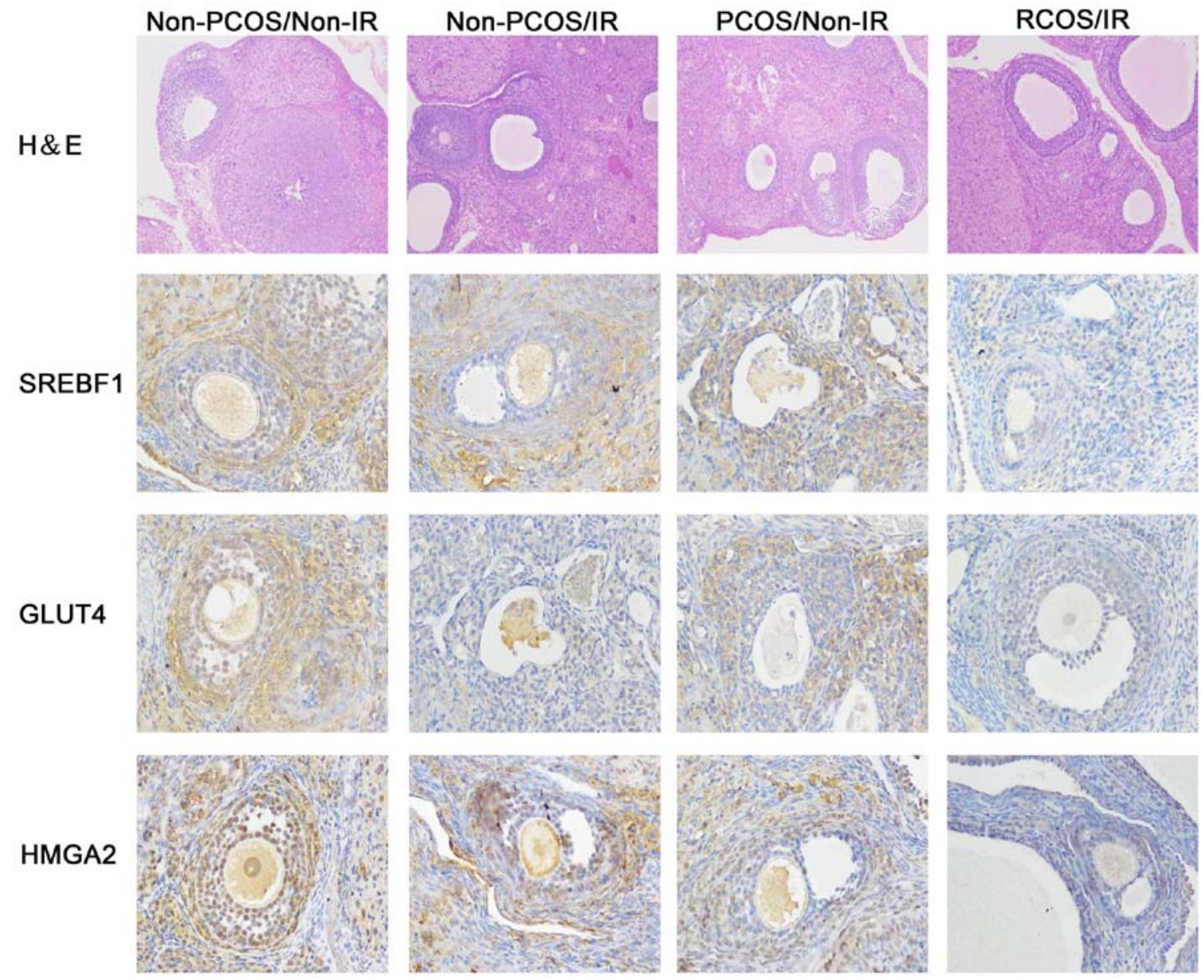

Figure 2. Hematoxylin and eosin (H\&E) staining and immunohistochemistry results for GLUT4, SREBF1 and HMGA2 in rat ovarian tissues from the non-PCOS/non-IR group, non-PCOS/IR group, PCOS/non-IR group and PCOS/IR group. Relative expression comparison by color (yellow to colorless, the nucleus was stained blue) was performed among four groups. Immunohistochemistry revealed that GLUT4, SREBF1 and HMGA2 were highly expressed in ovarian tissues from the non-PCOS/non-IR group, and their levels were significantly decreased in the PCOS/IR group (x100, magnification). PCOS, polycystic ovary syndrome; IR, insulin resistance.

SREBF1 and HMGA2, which validated the data by real-time PCR in Fig. 1. Consistently, obvious positive expression of GLUT4, SREBF1 and HMGA2 (yellow color) were found in non-PCOS/non-IR group compared to that in the other three groups. Immunohistochemistry data revealed that GLUT4, SREBF1 and HMGA2 were all highly expressed in the ovarian tissues of rats in the non-PCOS/non-IR group, however, their expression levels were significantly decreased in the PCOS/IR group (Fig. 2).

Excess extracellular glucose increases miR-33b-5p expression and reduces GLUT4, SREBF1 and HMGA2 expression. As shown in Fig. 3, excess extracellular glucose increased miR-33b-5p mRNA expression and reduced GLUT4, SREBF1, and HMGA2 expression in adipocytes (Fig. 3A-D). These findings were further supported by western blotting data (Fig. 3E-F). These results revealed that excess glucose can alter the level of miR-33b-5p expression, which in turn, may further alter the expression of mRNAs for GLUT4, SREBF1 and HMGA2 in adipocytes.

Excess extracellular insulin increases miR-33b-5p expression and reduces the levels of GLUT4, SREBF1 and HMGA2 expression. We also assessed the effect of extracellular insulin on miR-33b-5p, GLUT4, SREBF1 and HMGA2 expression in adipocytes. We found that insulin produced a similar effect as glucose; it upregulated miR-33b-5p levels and downregulated GLUT4, SREBF1 and HMGA2 mRNA and protein levels (Fig. 4). These results revealed that GLUT4, SREBF1 and HMGA2 expression in human adipocytes may also be regulated by changes in miR-33b-5p levels induced by excess insulin.

Downregulation of miR-33b-5p induces GLUT4, SREBF1 and HMGA2 expression. After determining that increased miR-33b-5p levels produced by treatment with either excess extracellular glucose or insulin suppressed GLUT4, SREBF1 and HMGA2 expression, we examined the effect of miR-33b-5p on GLUT4, SREBF1 and HMGA2 levels in adipocytes by reducing miR-33b-5p expression. Our data revealed that the expression levels miR-33b-5p gradually decreased as the concentration of miR-33b-5p inhibitor increased. In contrast, the GLUT4, SREBF1 and HMGA2 mRNA and protein levels increased in a concentration-dependent manner, suggesting that miR-33b-5p negatively regulates the levels of GLUT4, SREBF1 and HMGA2 (Fig. 5). 
A

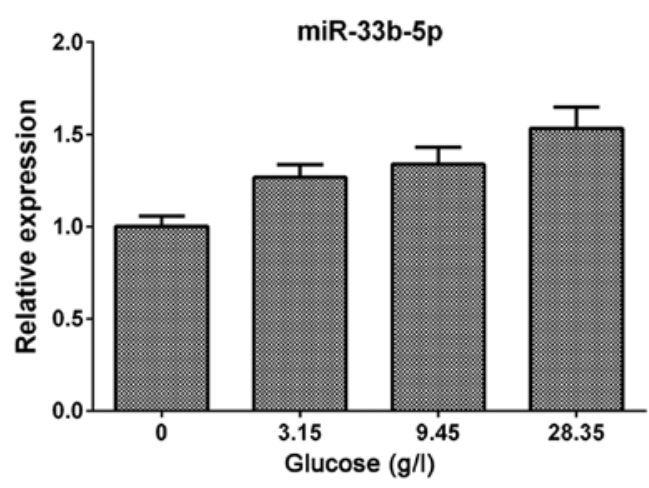

C

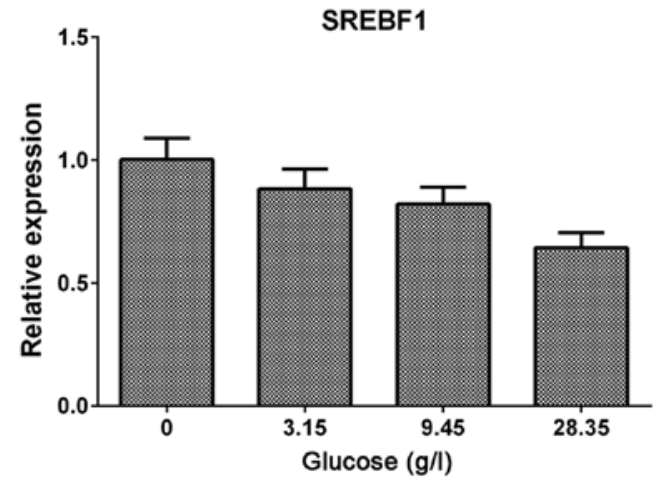

$E$

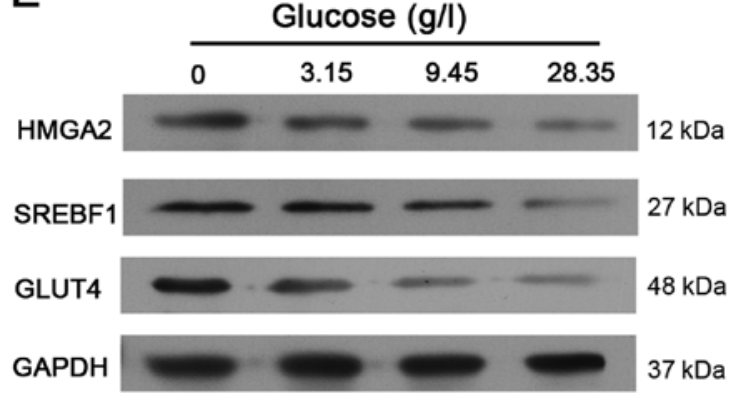

B

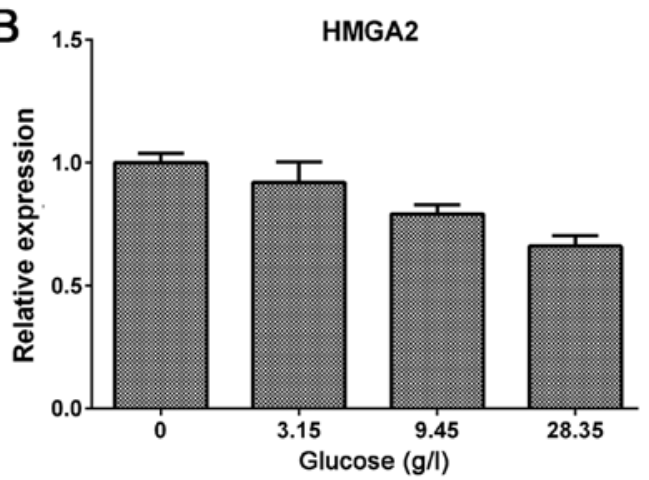

D

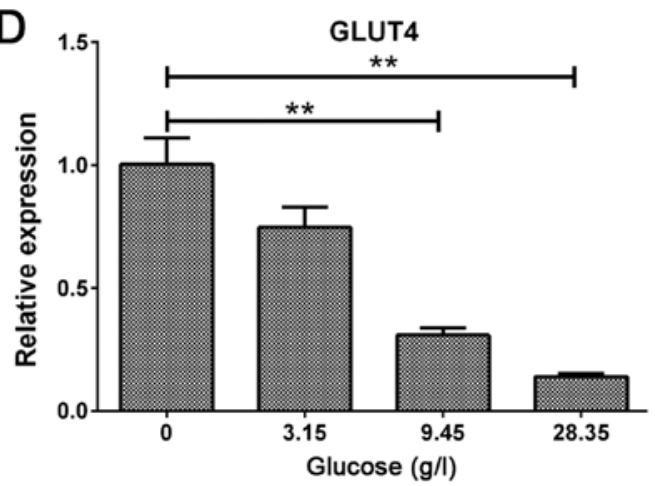

$\mathrm{F}$

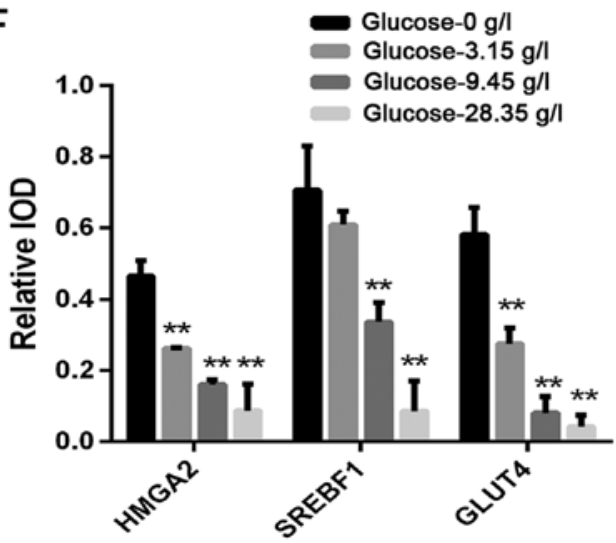

Figure 3. Changes in miR-33b-5p, GLUT4, SREBF1 and HMGA2 in glucose-treated adipocytes. (A-D) Changes in miR-33b-5p, GLUT4, SREBF1 and HMGA2 mRNA expression levels in adipocytes. (E-F) Western blotting results and quantification of these results revealing the GLUT4, SREBF1 and HMGA2 protein levels in the four groups treated with glucose at concentrations of $0,3.15,9.45$ and $28.35 \mathrm{~g} / 1$, respectively. ${ }^{* *} \mathrm{P}<0.01$.

The role of miR-33b-5p in regulating HMGA2, SREBF-1 and GLUT4 expression. Transient co-transfection of the SREBF1 5'-promoter luciferase vector with either the pcDNA3.0HMGA2 overexpression or empty plasmid revealed that pcDNA3.0-HMGA2 significantly increased luciferase activity in a dose-dependent manner, suggesting that HMGA2 could target SREBF1 and promote its expression (Fig. 6A). Chip data (Fig. 7A) revealed that HMGA2 directly binds to the 5'-promoter region of $S R E B F 1$ and promotes its expression.

Transientco-transfectionoftheGLUT45'-promoterluciferase vector with either the pcDNA3.0-HMGA2 overexpression or empty plasmid revealed that pcDNA3.0-HMGA2 also increased luciferase activity in a dose-dependent manner (Fig. 6B). Chip experiments also demonstrated that HMGA2 could bind to the 5'-promoter region of GLUT4 (Fig. 7B). These findings revealed that HMGA2 can directly bind to the 5'-promoter region of GLUT4 and promote its expression.

Transient co-transfection of the wild-type GLUT4 5'-promoter or mutant type vectors with either the pcDNA3.0SREBF1 overexpression or empty plasmid revealed that SREBF1 could significantly increase luciferase activity in the wild-type GLUT4 5'-promoter group (Fig. 6C). Chip experiments also demonstrated that SREBF1 could bind to the 5'-promoter region of GLUT4 (Fig. 7C). These findings indicated that SREBF1 can directly bind to the 5'-promoter region of GLUT4 and promote its expression.

Transient co-transfection of the wild-type luciferase HMGA2 3'-UTR and mutant vectors with either miR-33b-5p mimics or NC mimics into $293 \mathrm{~T}$ cells demonstrated direct binding of miR-33b-5p to the 3'-UTR of HMGA2, and resulted 
A

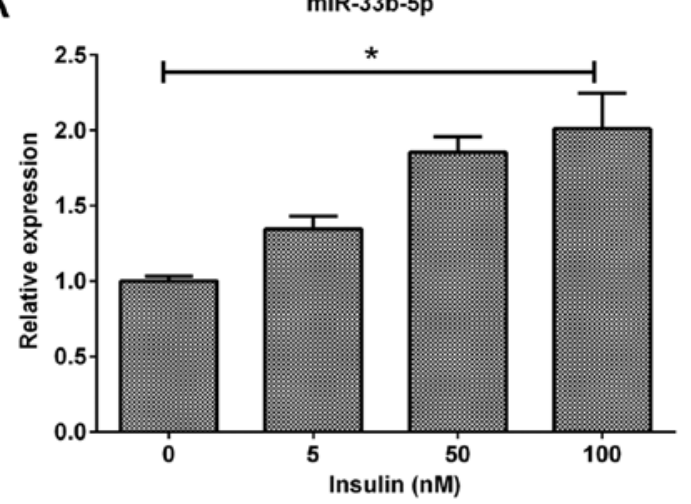

C

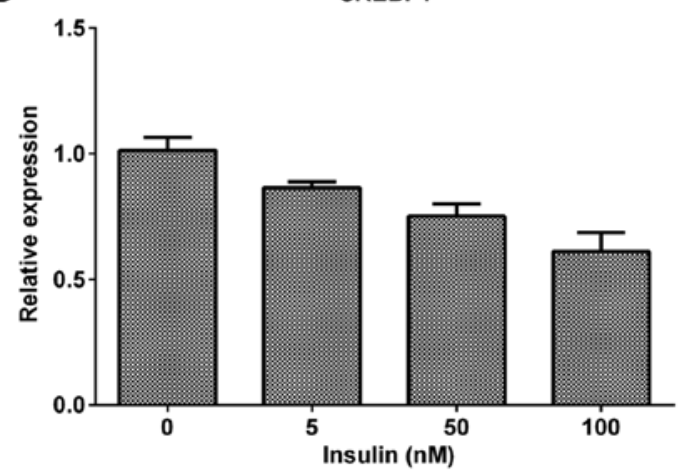

E

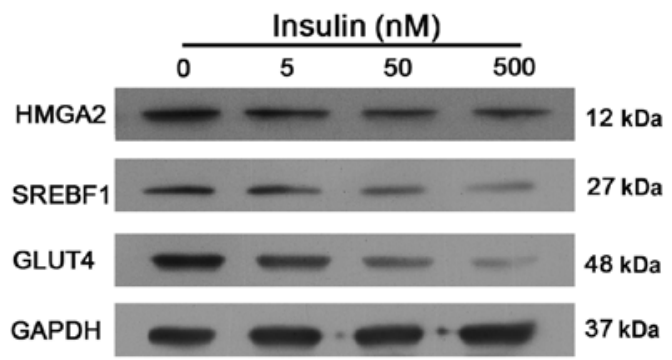

B

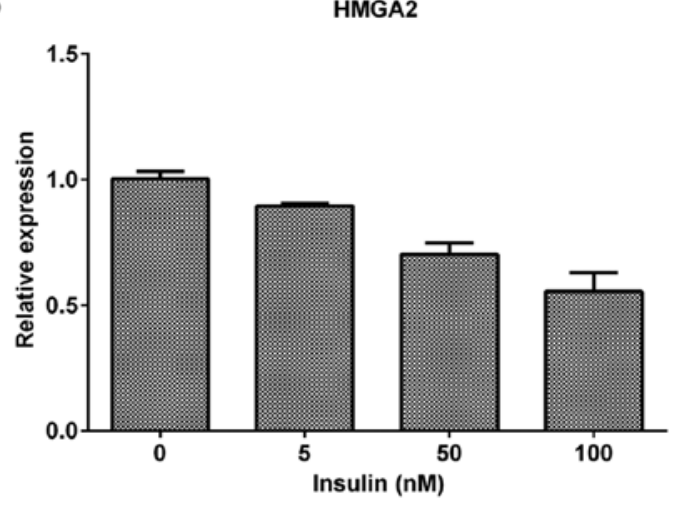

D

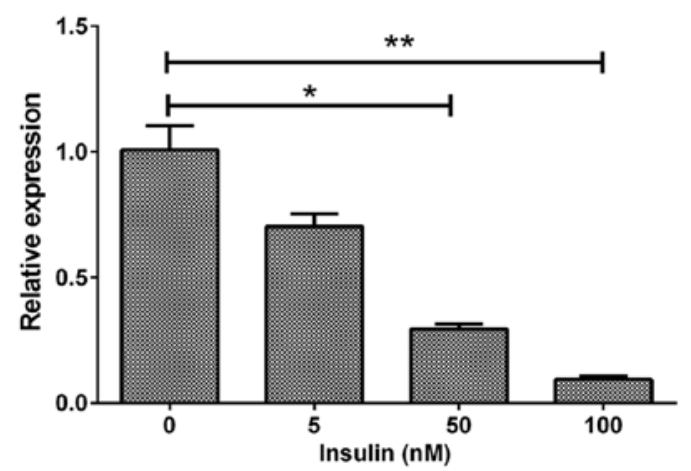

F

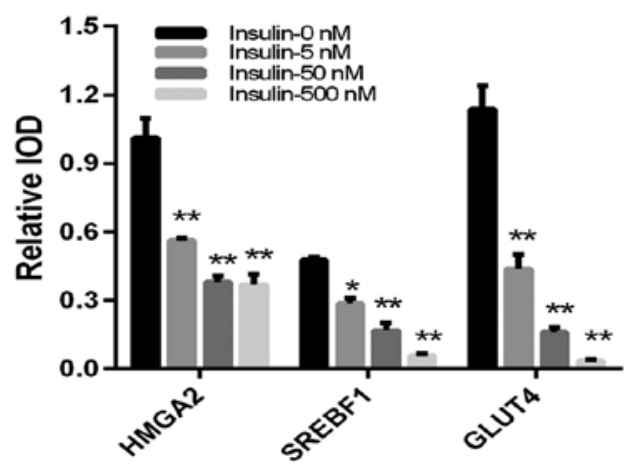

Figure 4. Insulin induces changes in miR-33b-5p, GLUT4, SREBF1 and HMGA2 in adipocytes. (A-D) Insulin induced changes in the miR-33b-5p, GLUT4, SREBF1 and HMGA2 mRNA levels in adipocyte cells. (E-F) Western blotting results and quantification of these results revealing the GLUT4, SREBF1 and HMGA2 protein levels in the four groups treated with insulin at concentrations of $0,5,50$ and $500 \mathrm{nM}$, respectively. ${ }^{*} \mathrm{P}<0.01$ and ${ }^{* * *} \mathrm{P}<0.01$.

in significant reductions in luciferase activity (Fig. 6D). These results revealed that miR-33b-5p could target the 3'-UTR of HMGA2 and inhibit its expression.

\section{Discussion}

The present study investigated the role of miR-33b-5p in PCOS, and particularly its role in regulating GLUT4. We determined that miR-33b-5p expression was higher in PCOS/IR rats, and correlated with SREBF1 and HMGA2 expression $(\mathrm{P}<0.05)$. Results of luciferase reporter assays and ChIP studies performed with adipocytes revealed that miR-33b-5p could target the 3'-UTR of HMGA2 and inhibit its expression. Moreover, HMGA2 could directly bind to the 5'-promoter region of GLUT4 and promote its expression, and could also promote SREBF1 expression. Finally, we determined that SREBF1 could directly bind to the 5'-promoter region of GLUT4 and promote its expression. Therefore, our study results demonstrated that miR-33b-5p was overexpressed in the ovarian tissues of PCOS/IR rats, and revealed that miR-33b-5p can inhibit GLUT4 by targeting HMGA2 during the development of insulin resistance in PCOS patients. Our results also indicated that HMGA2 and SREBF1 are important molecules involved in modulating GLUT4 expression.

miRNAs consist of a group of small non-coding RNAs, some of which play key roles in modulating the functions of human bodies $(25,34,35)$. Numerous miRNAs have been demonstrated to be involved in endocrine and metabolic diseases $(36,37)$, and several miRNAs (e.g., miR-93 and miR-145) have been reported to be involved in the pathogenesis of PCOS (27-31,38-42). miRNA-93 was initially revealed to play an important role in modulating GLUT4 expression, 

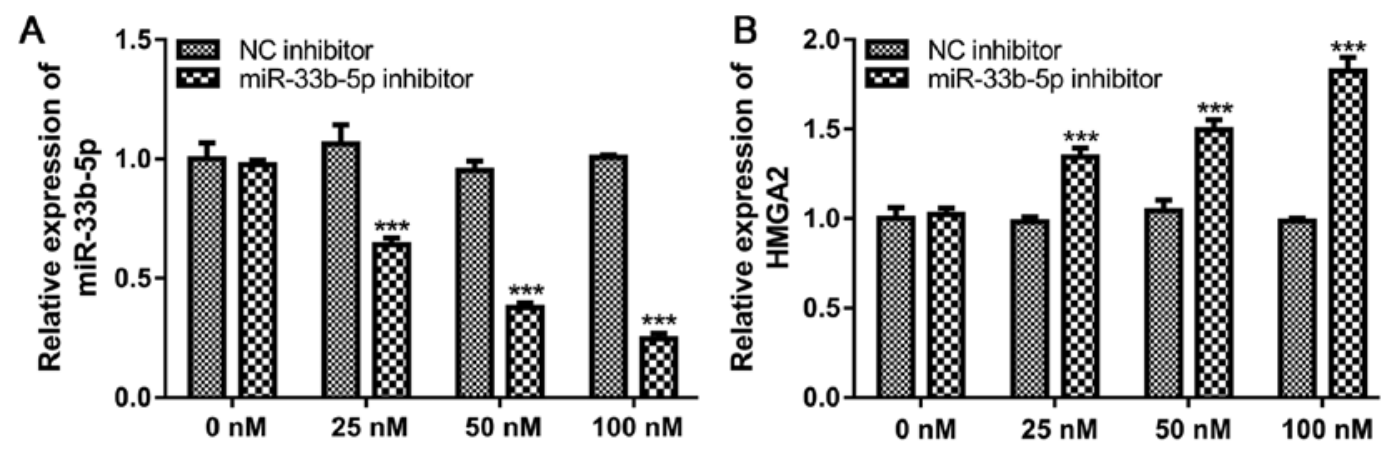

$$
\text { C }
$$
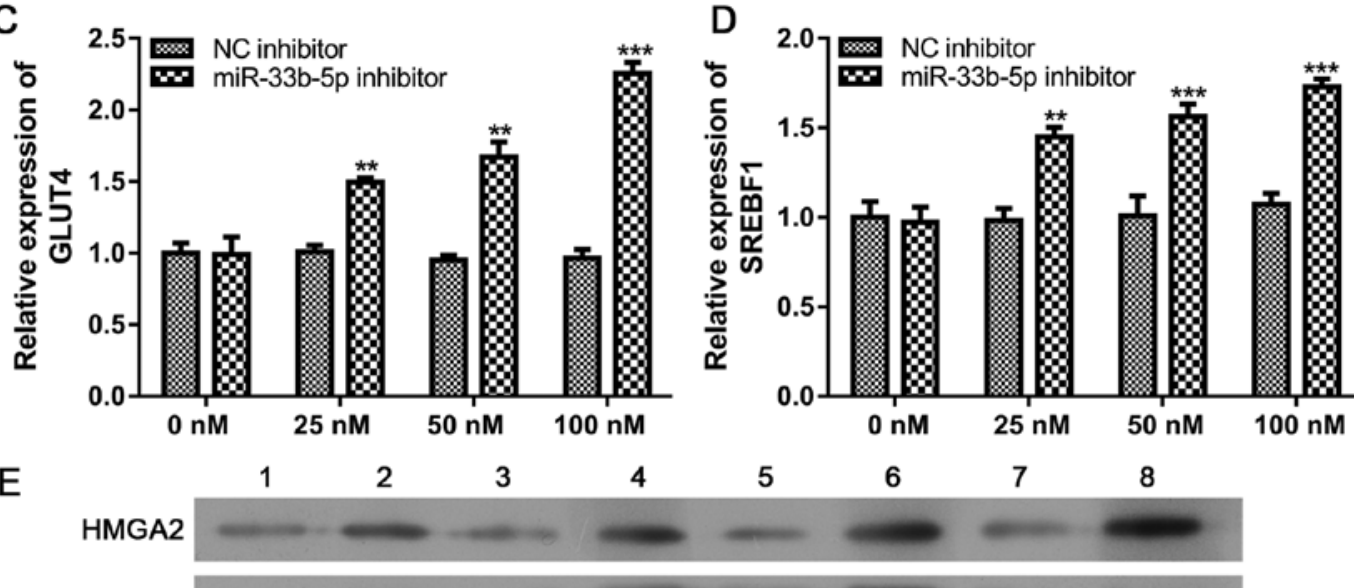

$\mathrm{E}$
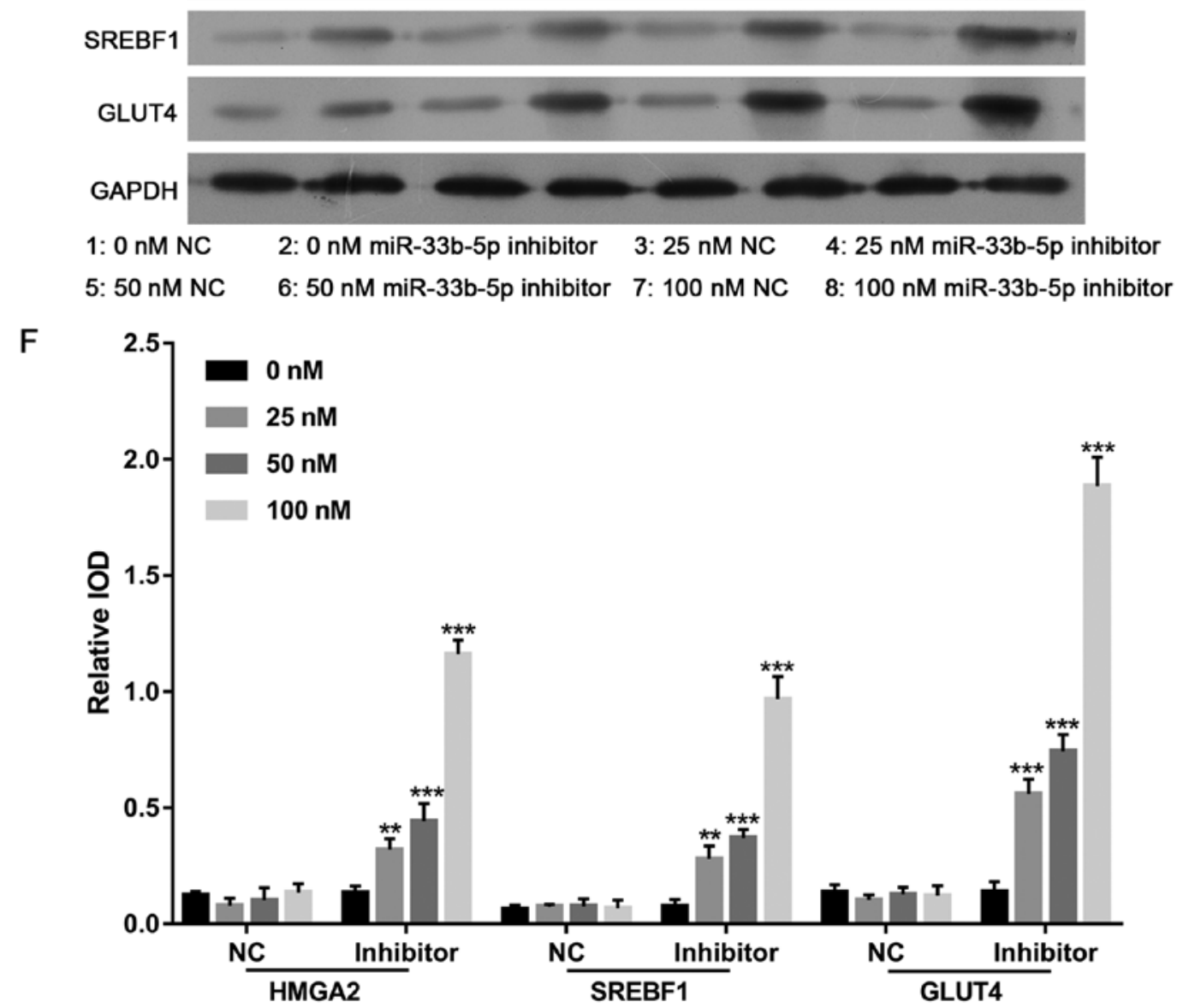

Figure 5. Effect of the miR-33b-5p inhibitor on miR-33b-5p, GLUT4, SREBF1 and HMGA2 in adipocytes (A-D) Levels of miR-33b-5p, GLUT4, SREBF1 and HMGA2 in the adipocytes. (E-F) Western blot results showing GLUT4, SREBF1 and HMGA2 protein levels in the eight groups treated with the control molecule or miR-33b-5p inhibitor at concentrations of $0,25,50$ and $500 \mathrm{nM}$, respectively. ${ }^{* *} \mathrm{P}<0.01$ and ${ }^{* * *} \mathrm{P}<0.001$, when compared to treatment with the control.

and thus be involved in the development of insulin resistance in PCOS patients (27). miR-145 was identified as a molecular target associated with granulosa cell dysfunction in PCOS, because it could inhibit IRS1 expression and the MAPK/ERK 
A
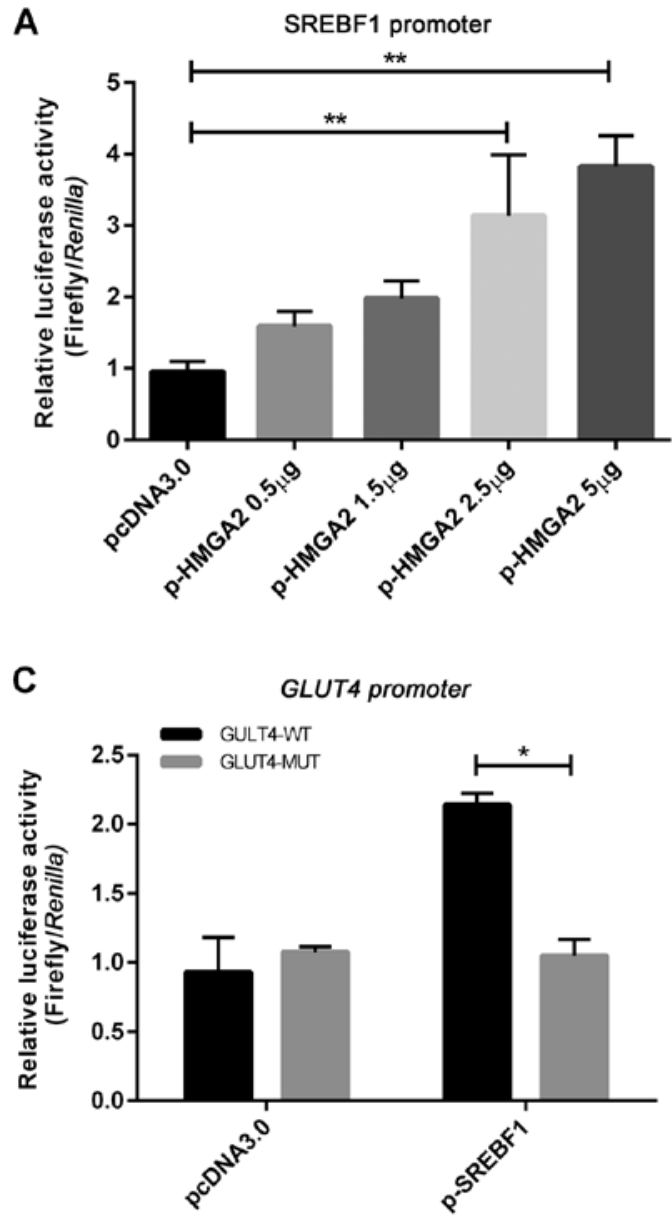

B

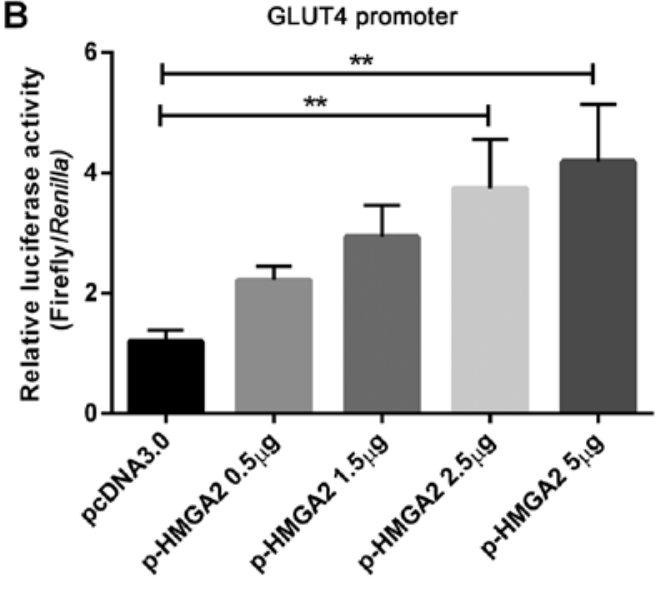

D

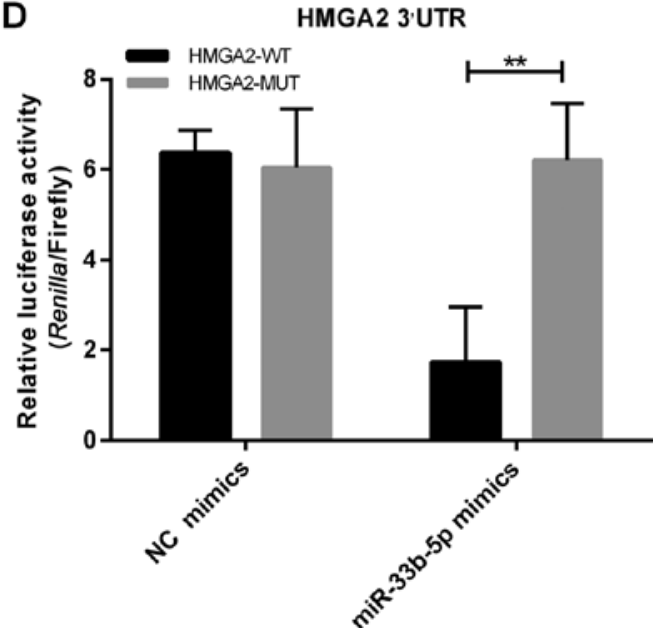

Figure 6. Findings from luciferase reporter assays. (A) pcDNA3.0-HMGA2 significantly increased luciferase activity of SREBF1 5'-promoter in a dose-dependent manner. (B) pcDNA3.0-HMGA2 also dose-dependently increased the luciferase activity of GLUT4 5'-promoter. (C) pcDNA3.0-SREBF1 significantly increased the luciferase activity of GLUT4 5'-promoter group. (D) Direct binding of miR-33b-5p to the 3'-UTR of HMGA2 resulted in significant reductions in luciferase activity. ${ }^{*} \mathrm{P}<0.05$ and ${ }^{* *} \mathrm{P}<0.01$.

A

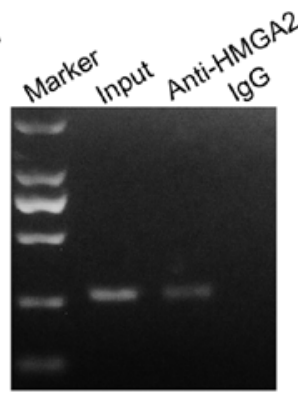

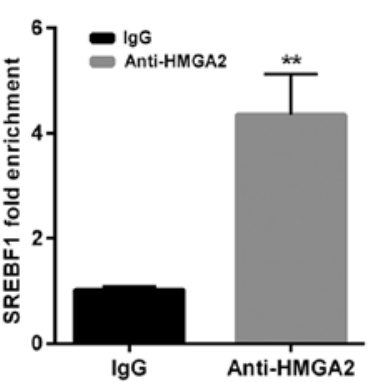

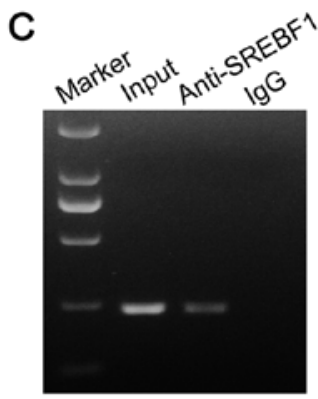

B
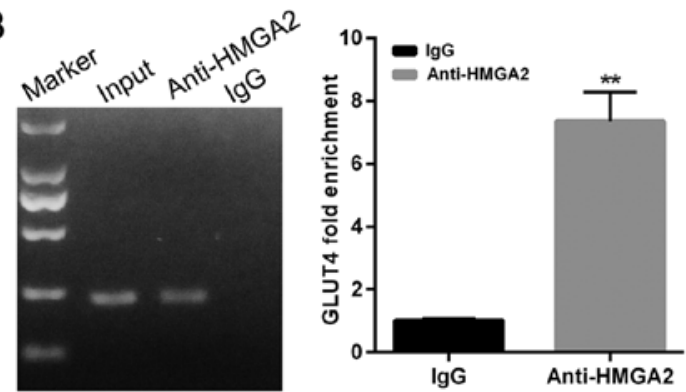

Figure 7. Outcomes from the ChIP experiments. (A) HMGA2 could bind to the 5'-promoter region of SREBF1. (B) HMGA2 could bind to the 5'-promoter region of GLUT4. (C) SREBF1 could bind to the 5'-promoter region of GLUT4. Left lane: representative ChIP images; right lane: quantification of PCR results. *** $<<0.01$. 
signaling pathways (43). The findings aforementioned revealed that some miRNAs indeed play key roles in the development of PCOS. In the present study, we found that miR-33b-5p was highly involved in the development of PCOS, and its overexpression was observed in our rat model of PCOS. The findings in our present study provide new additional evidence that miRNAs play key roles in the development of PCOS.

We further examined how miR-33b-5p affects GLUT4, SREBF1 and HMGA2 expression. Both excess extracellular glucose and insulin suppressed the levels of GLUT4, SREBF1 and HMGA2 by upregulating miR-33b-5p, whereas GLUT4, SREBF1 and HMGA2 levels became elevated when miR-33b-5p was inhibited, indicating the powerful effects that glucose and insulin exert in the regulation of GLUT4, SREBF1 and HMGA2 by miR-33b-5p. Insulin resistance is a main feature of PCOS, and can adversely affect a woman's health $(8,44,45)$. GLUT4 is strongly linked to the development of insulin resistance in type 2 diabetes or PCOS $(46,47)$. GLUT4 is a transporter molecule responsible for the uptake of glucose, and its dysfunction is observed in individuals with insulin resistance or PCOS (48). When blood glucose levels are low, GLUT4 is stored intracellularly in muscle and fat cells, preventing GLUT4 from reaching the cell surface and transporting glucose into cells (49). When blood glucose levels are high, GLUT4 is translocated to the cell surface after receiving an intracellular signal produced by insulin, resulting in glucose uptake (49). Therefore, adequate intracellular retention and a quick response to insulin stimulation are needed to maintain the function of GLUT4 in modulating glucose homeostasis. GLUT4 is currently regarded as an important molecule for regulating glucose homeostasis, and also a key factor involved in the development of insulin resistance (49). A previous study found decreased levels of GLUT4 expression in the endometrium of PCOS patients (13); however, the mechanism for that decrease is largely unclear. In the present study we found that GLUT4 expression could be regulated by HMGA2 and SREBF1, which offers a novel explanation for the aberrant expression of GLUT4 in PCOS patients. Our findings also provided some new promising therapeutic targets for PCOS, and suggest that increasing GLUT4 expression by inhibiting miR-33b-5p or increasing HMGA2 and SREBF1 levels may be a promising strategy for treating PCOS.

HMGA2 is a non-histone chromosomal protein with three DNA binding domains, and can act as a transcription regulating factor (50). HMGA2 can alter the structure of chromatin by forming a stereospecific complex with other proteins in the promoter or enhancer region of certain genes (51). It is also an essential component of the enhanceosome, and can promote the assembly of protein complexes and regulate the transcription of target genes (51). Previous studies have revealed that HMGA2 is associated with lipomas, indicating its roles in adipogenesis and insulin resistance $(14,15)$. In the present study, we demonstrated that miR-33b-5p was responsible for regulating HMGA2 and HMGA2 could regulate GLUT4 transcription. These findings explain the roles played by miR-33b-5p and GLUT4 in the pathogenesis of PCOS at a molecular level.

SREBF1 is a major regulator of genes involved in metabolic pathways and sterol biosynthesis, as it binds to the steroid regulatory element of the promoter or enhancer of those genes (16-18). Previous studies have revealed that SREBF1 is an important transcription factor that regulates certain genes related to lipid metabolism, which is also associated with insulin resistance $(52,53)$. In the present study, we determined that SREBF1 could directly regulate the target gene of GLUT4, which is a key gene involved in insulin resistance. We also determined that miR-33b-5p could target SREBPI and inhibit its expression. Moreover, we revealed that SREBP1 could regulate the expression of GLUT4 as a transcription factor, which further illustrates the key roles played by miR-33b-5p and GLUT4 in the development of PCOS.

In summary, the findings from this study revealed that miR-33b-5p was overexpressed in the ovarian tissues of PCOS rats with insulin resistance, and thus may play an important role in the development of insulin resistance in PCOS patients. miR-33b-5p could inhibit GLUT4 by targeting HMGA2 during the development of PCOS. Furthermore, HMGA2 and SREBF1 are important molecules involved in modulating GLUT4 expression. These findings enhanced our understanding of the molecular mechanisms that contribute to insulin resistance during the development of PCOS, and may assist in finding new therapeutic targets for PCOS. Future studies may determine whether it is feasible to treat PCOS by targeting miR-33b-5p.

\section{Acknowledgements}

Not applicable.

\section{Funding}

The present study was supported by the Natural Science Foundation of Hubei Province (no. 2014CFB251).

\section{Availability of data and materials}

The datasets used during the present study are available from the corresponding author upon reasonable request.

\section{Authors' contributions}

YY, HJ and XZY conceived and designed the study. YY, HJ and LX performed the experiments. YY and XZY wrote the paper. All authors read and approved the manuscript and agree to be accountable for all aspects of the research in ensuring that the accuracy or integrity of any part of the work are appropriately investigated and resolved.

\section{Ethics approval and consent to participate}

All tissue specimens were obtained with permission from the Affiliated Hospital of Hubei University. All participants have read and signed the written informed consent.

\section{Consent for publication}

All participants have read and signed the written informed consent for the publication.

\section{Competing interests}

The authors declare that they have no competing interests. 


\section{References}

1. Azziz R, Carmina E, Chen Z, Dunaif A, Laven JS, Legro RS, Lizneva D, Natterson-Horowtiz B, Teede HJ, Yildiz BO Polycystic ovary syndrome. Nat Rev Dis Primers 2: 16057, 2016.

2. Barber TM, Dimitriadis GK, Andreou A and Franks S: Polycystic ovary syndrome: Insight into pathogenesis and a common association with insulin resistance. Clin Med 16: 262-266, 2016.

3. Lauritsen MP, Bentzen JG, Pinborg A, Loft A, Forman JL, Thuesen LL, Cohen A, Hougaard DM and Nyboe Andersen A: The prevalence of polycystic ovary syndrome in a normal population according to the Rotterdam criteria versus revised criteria including anti-Mullerian hormone. Hum Reprod 29: 791-801, 2014.

4. Li R, Zhang Q, Yang D, Li S, Lu S, Wu X, Wei Z, Song X, Wang X, Fu S, et al: Prevalence of polycystic ovary syndrome in women in China: A large community-based study. Hum Reprod 28: 2562-2569, 2013.

5. Marciniak A, Nawrocka Rutkowska J, Brodowska A, Wiśniewska B and Starczewski A: Cardiovascular system diseases in patients with polycystic ovary syndrome - the role of inflammation process in this pathology and possibility of early diagnosis and prevention. Ann Agric Environ Med 23: 537-541, 2016.

6. Merz CNB, Shaw LJ, Azziz R, Stanczyk FZ, Sopko G, Braunstein GD, Kelsey SF, Kip KE, Cooper-DeHoff RM, Johnson BD, et al: Cardiovascular disease and 10-year mortality in postmenopausal women with clinical features of polycystic ovary syndrome. J Womens Health 25: 875-881, 2016.

7. Velija-Asimi Z, Burekovic A, Dujic T, Dizdarevic-Bostandzic A and Semiz S: Incidence of prediabetes and risk of developing cardiovascular disease in women with polycystic ovary syndrome. Bosn J Basic Med Sci 16: 298-306, 2016.

8. Jeanes YM and Reeves S: Metabolic consequences of obesity and insulin resistance in polycystic ovary syndrome: Diagnostic and methodological challenges. Nutr Res Rev 30: 97-105, 2017.

9. Morgan BJ, Chai SY and Albiston AL: GLUT4 associated proteins as therapeutic targets for diabetes. Recent Pat Endocr Metab Immune Drug Discov 5: 25-32, 2011.

10. Govers R: Cellular regulation of glucose uptake by glucose transporter GLUT4. Adv Clin Chem 66: 173-240, 2014.

11. Johansson J, Feng Y, Shao R, Lönn M, Billig H and StenerVictorin E: Intense electroacupuncture normalizes insulin sensitivity, increases muscle GLUT4 content, and improves lipid profile in a rat model of polycystic ovary syndrome. Am J Physiol Endocrinol Metab 299: E551-E559, 2010.

12. Mozzanega B, Mioni R, Granzotto M, Chiarelli S, Xamin N, Zuliani L, Sicolo N, Marchesoni D and Vettor R: Obesity reduces the expression of GLUT4 in the endometrium of normoinsulinemic women affected by the polycystic ovary syndrome. Ann NY Acad Sci 1034: 364-374, 2004.

13. Zhai J, Liu CX, Tian ZR, Jiang QH and Sun YP: Effects of metformin on the expression of GLUT4 in endometrium of obese women with polycystic ovary syndrome. Biol Reprod 87: 29, 2012.

14. Xi Y, Shen W, Ma L, Zhao M,Zheng J, Bu S, Hino S and Nakao M: HMGA 2 promotes adipogenesis by activating C/EBP $\beta$-mediated expression of PPAR $\gamma$. Biochem Biophys Res Commun 472 617-623, 2016

15. Yuan Y, Xi Y, Chen J, Zhu P, Kang J, Zou Z, Wang F and Bu S: STAT3 stimulates adipogenic stem cell proliferation and cooperates with HMGA2 during the early stage of differentiation to promote adipogenesis. Biochem Biophys Res Commun 482: 1360-1366, 2017

16. Oishi Y, Spann NJ, Link VM, Muse ED, Strid T, Edillor C, Kolar MJ, Matsuzaka T, Hayakawa S, Tao J, et al: SREBP1 contributes to resolution of pro-inflammatory TLR4 signaling by reprogramming fatty acid metabolism. Cell Metab 25: 412-427, 2017.

17. Shafiee MN, Mongan N, Seedhouse C, Chapman C, Deen S, Abu J and Atiomo W: Sterol regulatory element binding protein-1 $(S R E B P 1)$ gene expression is similarly increased in polycystic ovary syndrome and endometrial cancer. Acta Obstet Gynecol Scand 96: 556-562, 2017

18. Yang L, Chen J, Li Y, Wang Y, Liang S, Shi Y, Shi S and Xu Y: Association between $S C A P$ and $S R E B F 1$ gene polymorphisms and metabolic syndrome in schizophrenia patients treated with atypical antipsychotics. World J Biol Psychiatry 17: 467-474, 2016.
19. Flowers E, Froelicher ES and Aouizerat BE: MicroRNA regulation of lipid metabolism. Metabolism 62: 12-20, 2013.

20. Ebert MS and Sharp PA: Roles for microRNAs in conferring robustness to biological processes. Cell 149: 515-524, 2012.

21. Bartel DP: MicroRNAs: Target recognition and regulatory functions. Cell 136: 215-233, 2009.

22. Yates LA, Norbury CJ and Gilbert RJC: The long and short of microRNA. Cell 153: 516-519, 2013.

23. Osmai M, Osmai Y, Bang-Berthelsen $\mathrm{CH}$, Pallesen EM, Vestergaard AL, Novotny GW, Pociot F and Mandrup-Poulsen T: MicroRNAs as regulators of beta-cell function and dysfunction. Diabetes Metab Res Rev 32: 334-349, 2016.

24. Vienberg S, Geiger J, Madsen S and Dalgaard LT: MicroRNAs in metabolism. Acta Physiol 219: 346-361, 2017.

25. Cruz KJ, de Oliveira AR, Morais JB, Severo JS and Marreiro DD: Role of microRNAs on adipogenesis, chronic low-grade inflammation, and insulin resistance in obesity. Nutrition 35: 28-35, 2017.

26. Williams MD and Mitchell GM: MicroRNAs in insulin resistance and obesity. Exp Diabetes Res 2012: 484696, 2012

27. Chen YH, Heneidi S, Lee JM, Layman LC, Stepp DW, Gamboa GM, Chen BS, Chazenbalk G and Azziz R: miRNA-93 inhibits GLUT4 and is overexpressed in adipose tissue of polycystic ovary syndrome patients and women with insulin resistance. Diabetes 62: 2278-2286, 2013.

28. Hossain MM, Cao M, Wang Q, Kim JY, Schellander K, Tesfaye D and Tsang BK: Altered expression of miRNAs in a dihydrotestosterone-induced rat PCOS model. J Ovarian Res 6: 36, 2013.

29. Ilie IR and Georgescu CE: Polycystic ovary syndrome-epigenetic mechanisms and aberrant microRNA. Adv Clin Chem 71:25-45, 2015.

30. Jiang L, Huang J, Li L, Chen Y, Chen X, Zhao X and Yang D: MicroRNA-93 promotes ovarian granulosa cells proliferation through targeting CDKN1A in polycystic ovarian syndrome. J Clin Endocrinol Metab 100: E729-E738, 2015.

31. Sørensen AE, Udesen PB, Wissing ML, Englund AL and Dalgaard LT: MicroRNAs related to androgen metabolism and polycystic ovary syndrome. Chem Biol Interact 259: 8-16, 2016.

32. Yin M, Wang X, Yao G, Lü M, Liang M, Sun Y and Sun F: Transactivation of microRNA-320 by microRNA-383 regulates granulosa cell functions by targeting E2F1 and SF-1 proteins. J Biol Chem 289: 18239-18257, 2014.

33. Poretsky L, Clemons J and Bogovich K: Hyperinsulinemia and human chorionic gonadotropin synergistically promote the growth of ovarian follicular cysts in rats. Metabolism 41: 903-910, 1992.

34. Adams BD, Parsons C, Walker L, Zhang WC and Slack FJ: Targeting noncoding RNAs in disease. J Clin Invest 127: 761-771, 2017.

35. Yee D, Coles MC and Lagos D: microRnAs in the lymphatic endothelium: Master regulators of lineage plasticity and inflammation. Front Immunol 8: 104, 2017.

36. Butz H, Kinga N, Racz K and Patocs A: Circulating miRNAs as biomarkers for endocrine disorders. J Endocrinol Invest 39: 1-10, 2016.

37. Derghal A, Djelloul M, Trouslard J and Mounien L: An emerging role of micro-RNA in the effect of the endocrine disruptors. Front Neurosci 10: 318, 2016

38. Jiang L, Huang J, Chen Y, Yang Y, Li R, Li Y, Chen X and Yang D: Identification of several circulating microRNAs from a genome-wide circulating microRNA expression profile as potential biomarkers for impaired glucose metabolism in polycystic ovarian syndrome. Endocrine 53: 280-290, 2016.

39. Li C, Chen L, Zhao Y, Chen S, Fu L, Jiang Y, Gao S, Liu Z, Wang F, Zhu X, et al: Altered expression of miRNAs in the uterus from a letrozole-induced rat PCOS model. Gene 598: 20-26, 2017.

40. Salimi-Asl M, Mozdarani H and Kadivar M: Up-regulation of miR-21 and 146a expression and increased DNA damage frequency in a mouse model of polycystic ovary syndrome (PCOS). Bioimpacts 6: 85-91, 2016.

41. Sørensen AE, Wissing ML, Englund ALM and Dalgaard LT: MicroRNA species in follicular fluid associating with polycystic ovary syndrome and related intermediary phenotypes. J Clin Endocrinol Metab 101: 1579-1589, 2016.

42. Zhang CL, Wang H, Yan CY, Gao XF and Ling XJ: Deregulation of RUNX2 by miR-320a deficiency impairs steroidogenesis in cumulus granulosa cells from polycystic ovary syndrome (PCOS) patients. Biochem Biophys Res Commun 482: 1469-1476, 2017. 
43. Cai G, Ma X, Chen B, Huang Y, Liu S, Yang H and Zou W: MicroRNA-145 negatively regulates cell proliferation through targeting IRS1 in isolated ovarian granulosa cells from patients with polycystic ovary syndrome. Reprod Sci 24: 902-910, 2017.

44. Abruzzese GA, Cerrrone GE, Gamez JM, Graffigna MN, Belli S, Lioy G, Mormandi E, Otero P, Levalle OA and Motta AB: Lipid accumulation product (LAP) and visceral adiposity index (VAI) as markers of insulin resistance and metabolic associated disturbances in young argentine women with polycystic ovary syndrome. Horm Metab Res 49: 23-29, 2017.

45. Temur M, Yılmaz Ö, Aksun S, Calan M, Özün Özbay P, Kumbasar S and Sever E: The relationship of urocortin-2 with insulin resistance patients having PCOS. Gynecol Endocrinol 33: 124-127, 2017.

46. Boden G, Homko C, Barrero CA, Stein TP, Chen X, Cheung P, Fecchio C, Koller S and Merali S: Excessive caloric intake acutely causes oxidative stress, GLUT4 carbonylation, and insulin resistance in healthy men. Sci Transl Med 7: 304re7, 2015.

47. Fazakerley DJ, Naghiloo S, Chaudhuri R, Koumanov F, Burchfield JG, Thomas KC, Krycer JR, Prior MJ, Parker BL, Murrow BA, et al: Proteomic analysis of GLUT4 storage vesicles reveals tumor suppressor candidate 5 (TUSC5) as a novel regulator of insulin action in adipocytes. J Biol Chem 290: 23528-23542, 2015
48. Belman JP, Bian RR, Habtemichael EN, Li DT, Jurczak MJ, Alcázar-Román A, McNally LJ, Shulman GI and Bogan JS: Acetylation of TUG protein promotes the accumulation of GLUT4 glucose transporters in an insulin-responsive intracellular compartment. J Biol Chem 290: 4447-4463, 2015.

49. Leto D and Saltiel AR: Regulation of glucose transport by insulin: Traffic control of GLUT4. Nat Rev Mol Cell Biol 13: 383-396, 2012

50. Rowe RG, Wang LD, Coma S, Han A, Mathieu R, Pearson DS, Ross S, Sousa P, Nguyen PT, Rodriguez A, et al: Developmental regulation of myeloerythroid progenitor function by the Lin28b-let-7-Hmga2 axis. J Exp Med 213: 1497-1512, 2016.

51. Fedele M, Palmieri D and Fusco A: HMGA2: A pituitary tumour subtype-specific oncogene? Mol Cell Endocrinol 326: 19-24, 2010.

52. Han J, Li E, Chen L, Zhang Y, Wei F, Liu J, Deng H and Wang Y: The CREB coactivator CRTC2 controls hepatic lipid metabolism by regulating SREBP1. Nature 524: 243-246, 2015.

53. Gorgani-Firuzjaee S and Meshkani R: SH2 domain-containing inositol 5-phosphatase (SHIP2) inhibition ameliorates high glucose-induced de-novo lipogenesis and VLDL production through regulating AMPK/mTOR/SREBP1 pathway and ROS production in HepG2 cells. Free Radic Biol Med 89: 679-689, 2015. 